\title{
Efecto de la estequiometria y de la temperatura de cocción en el desarrollo de la fase $\mathrm{Al}_{2} \mathrm{TiO}_{5}$
}

\author{
A. P. FONSECA ${ }^{(1)}$, J. BAPTISTA $^{(2)}$ \\ (1) Universidade do Vale do Paraíba - Instituto de Pesquisa e Desenvolvimento \\ CEP: 12.244-000, São José dos Campos, SP, Brasil
}

\begin{abstract}
El Titanato de Aluminio $\left(\mathrm{Al}_{2} \mathrm{TiO}_{5}\right)$ debido a su bajo coeficiente de expansión térmica, elevada refractariedad y baja mojabilidad por metales no ferrosos fundidos, es un material prometedor en la industria del Aluminio para aplicaciones donde se exige

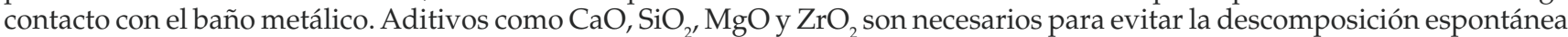
del $\mathrm{Al}_{2} \mathrm{TiO}_{5}$ en sus óxidos constituyentes a temperaturas inferiores a $1300^{\circ} \mathrm{C}$. En este trabajo, se ha investigado el efecto de la estequiometría y de la temperatura de sinterización en el desarrollo y estabilidad de la fase $\mathrm{Al}_{2} \mathrm{TiO}_{5}$. Se ha encontrado que un exceso de Óxido de Titanio $\left(\mathrm{TiO}_{2}\right)$ es beneficioso en la producción de $\mathrm{Al}_{2} \mathrm{TiO}_{5}$ puro y estable.
\end{abstract}

\section{Palabras-claves: Titanato de Aluminio, composición no-estequiométrica.}

\section{Effect of stoichiometry and sintering temperature in the development of $\mathrm{Al}_{2} \mathrm{TiO}_{5}$ phase}

Aluminum Titanate $\left(\mathrm{Al}_{2} \mathrm{TiO}_{5}\right)$ due to its low termal expansion coefficient, low wettability and high refractoriness is a promissing refractory ceramic to be in contact with non ferrous metals, specially molten aluminum. Controlled amounts of $\mathrm{CaO}, \mathrm{SiO}_{2}, \mathrm{MgO}$ and $\mathrm{ZrO}_{2}$ are normally added in order to avoid expontaneous decomposition, lower than $1300^{\circ} \mathrm{C}$, into its constituint oxides. In this work through the adoption of a new approach, it was investigated the $\mathrm{Al}_{2} \mathrm{TiO}_{5}$ phase development and stabilization with respect to stoichiometry and firing temperature. It was found that an excess of Titania $\left(\mathrm{TiO}_{2}\right)$ helps the development of a stable and purer Aluminum Titanate phase.

Key Words: Aluminum Titanate, non stoichiometry composition.

\section{INTRODUCCIÓN}

Recientemente el Titanato de Aluminio ha despertado gran interés en la industria de metales no ferrosos, particularmente en la industria del Aluminio, debido principalmente a que no es mojado por este metal en estado fundido. Además el bajo coeficiente de expansión térmica de los compactos policristalinos de este confere les una excelente resistencia al choque térmico, permitiendo también que el material sea utilizado en aplicaciones estructurales a elevadas temperaturas [1, 2].

El Titanato de Aluminio $\left(\mathrm{Al}_{2} \mathrm{TiO}_{5}\right)$ puede obtenerse por reacción en estado sólido de una mezcla equimolar entre Alúmina $\left(\mathrm{Al}_{2} \mathrm{O}_{3}\right)$ y Óxido de Titanio $\left(\mathrm{TiO}_{2}\right)$ a temperaturas superiores a $1300^{\circ} \mathrm{C}$. Este processo de sinterización reactiva origina un material poroso, conteniendo casi siempre Alúmina y Óxido de Titanio sin reaccionar $[2,3,4]$.

El bajo coeficiente de expansión térmica presentado por los compactos de $\mathrm{Al}_{2} \mathrm{TiO}_{5}$ se debe a la pronunciada anisotropía de los cirstales de $\mathrm{Al}_{2} \mathrm{TiO}_{5}$, ésto significa que dos ejes cristalinos tienen coeficiente de expansión térmica positivos y un tercero con coeficiente de expansión térmica negativo, lo que origina un microagrietamiento inter y intracristalino [2] en los compactos com un elevado tamaño de partícula. Este microagrietamiento puede ser minimizado controlándo el tamaño de grano, reduciéndolo hasta un valor inferior al crítico estimado entre 1 y $2 \mu \mathrm{m}$ [5].

La reacción de Alúmina y Óxido de Titanio para formar el Titanato de Aluminio tiene lugar en la interface $\mathrm{Al}_{2} \mathrm{O}_{3} / \mathrm{Al}_{2} \mathrm{TiO}_{5}$, por difusión del ión $\mathrm{Ti}^{+4}$ a través de los granos de $\mathrm{Al}_{2} \mathrm{TiO}_{5}$ ya formados. La incorporación de hierro a la red cristalina del Titanato de Aluminio origina defectos, que aumentan considerablemente la velocidad de difusión del ión $\mathrm{Ti}^{+4}$ [2]. Este hecho posibilita una reacción completa y la formación de un Titanato más denso y estable en relación a la descomposición a temperaturas entre $800-1300^{\circ} \mathrm{C}$, ya que la presencia de Alúmina residual actúa como núcleo de descomposición. La descomposición térmica del Titanato de Aluminio se ha controlado mediante la adición de porcentajes elevados de $\mathrm{CaO}, \mathrm{MgO}$, $\mathrm{SiO}_{2}, \mathrm{ZrO}_{2}[1,2,3,4]$, lo que también afecta a las características termomecánicas.

Teniendo en cuenta las limitaciones presentadas por los materiales de $\mathrm{Al}_{2} \mathrm{TiO}_{5}$ con aditivos, este trabajo procura adoptar un nuevo enfoque, investigando los efectos de la estequiometría y de la temperatura del processo de sinterización-reactiva en la obtención de una fase más pura y estable.

\section{PARTE EXPERIMENTAL}

Para la obtención de los materiales de Titanato de Aluminio $\left(\mathrm{Al}_{2} \mathrm{TiO}_{5}\right)$, se utilizó como óxidos precursores Óxido de Titanio $\left(\mathrm{TiO}_{2}\right.$ - Rutilo 99,5\%) y Alúmina $\left(\mathrm{Al}_{2} \mathrm{O}_{3}\right)$ calcinada ALCOA APC 3017-SG. Se hace preparado tres composiciones: estequiométrica (E), rica en Alúmina (A - 57,8\% $\mathrm{mol} \mathrm{Al}_{2} \mathrm{O}_{3}$ ) y 
rica en Óxido de Titanio ( $\mathrm{T}-56 \% \mathrm{~mol} \mathrm{TiO}_{2}$ ). Las composiciones representadas en el diagrama de fases de la Figura 1, se hace homogenizado en um molino de bolas con agua destilada, durante 6 horas. Posteriormente se hace secado a $110^{\circ} \mathrm{C}$, desagregado y tamizado através de um tamiz de 100 mallas, posteriormente se hace prensado en forma de barras de $(5 \times 10 \times 70) \mathrm{mm}$ a ena presión de $100 \mathrm{MPa}$. Las temperaturas de cocción fueron 1350,1400 y $1450^{\circ} \mathrm{C}$ por 3 horas.

Después de la sinterización se hace determinado las propiedades físicas seguientes: densidad aparente, porosidad aparente y variación dimensional lineal. El porcentaje de la fase Titanato de Aluminio $\left(\mathrm{Al}_{2} \mathrm{TiO}_{5}\right)$ así como de las fases $\mathrm{Al}_{2} \mathrm{O}_{3}$ y $\mathrm{TiO}_{2}$ sin reaccionar se há determinado de un modo semi-cuantitativo, mediante la comparación de la área (altura vezes el ancho a la media altura) del pico mas intenso obtidos de $\mathrm{Al}_{2} \mathrm{TiO}_{5}(020)$ a los picos mas intensos del $\mathrm{Al}_{2} \mathrm{O}_{3}(113)$ y $\mathrm{TiO}_{2}$ (110), de cada uno de los difractogramas en las diferentes temperaturas de sinterización.

Se há estudiado la estabilidad térmica del $\mathrm{Al}_{2} \mathrm{TiO}_{5}$ para las tres combinaciones de composición y temperatura de cocción. Para ésto las muestras fueron sometidas a un tratamiento térmico a $1100,1200 \mathrm{y} 1300^{\circ} \mathrm{C}$ durante $4 \mathrm{~h}$ y posteriormente a un análisis de difracción de rayos- $X$ usando el mismo procedimiento semi-cuantitativo descrito previamente.

\section{RESULTADOS}

La Figura 2 presenta los valores de densidad aparente para las tres muestras estudiadas de composición estequiométrica (E), rica en Alúmina (A) y rica en Óxido de Titanio (T), tratadas térmicamente entre 1350 e $1450^{\circ} \mathrm{C}$.

Las Figuras 3 y 4 presentan el análisis comparativo porcentual de las fases $\mathrm{Al}_{2} \mathrm{O}_{3}$ y TiO $\mathrm{Tin}_{2}$ sin reaccionar en las tres muestras estudiadas tratadas en diferentes temperaturas.

Las Figuras 5 y 6 presentan el análisis porcentual comparativo de las fases encontradas en la muestra de composición estequiométrica sinterizada a $1400^{\circ} \mathrm{C}(\mathrm{E}-1400)$ y composición con exceso de Óxido de Titanio sinterizada a $1400^{\circ} \mathrm{C}$ (T-1400) y a $1450^{\circ} \mathrm{C}(\mathrm{T}-1450)$, después de un tratamiento térmico a 1100 , 1200 y $1300^{\circ} \mathrm{C}$.

La Figura 7 presenta el difractograma de la composición con exceso de Óxido de Titanio tratada a $1400^{\circ} \mathrm{C}$.

\section{DISCUSIÓN}

Los valores de densidad aparente obtenidos para las composiciones estequiométrica (E), con exceso de Alúmina (A) y con exceso de Óxido de Titanio (T) presentan un comportamiento similar, esto significa que al aumentar la temperatura de 1350 a $1400^{\circ} \mathrm{C}$ tiene lugar una mayor densificación. A la temperatura de $1450^{\circ} \mathrm{C}$ tiene lugar una disminución acentuada de la densidad aparente, cuyos valores se aproximan a los obtenidos a $1350^{\circ} \mathrm{C}$. Este hecho puede ser consecuencia del crecimiento de grano de la fase $\mathrm{Al}_{2} \mathrm{TiO}_{5}$ que esta acompañado de un microagrietamiento intenso. Se puede notar también en la Figura 2 que la composición que mejor sinteriza a $1400^{\circ} \mathrm{C}$ es la composición rica en Alúmina (composición A).

El análisis porcentual comparativo de las fases Alúmina y Óxido de Titanio sin reaccionar presentado en las Figuras 3 y 4 indica la necesidad de un exceso de Óxido de Titanio para

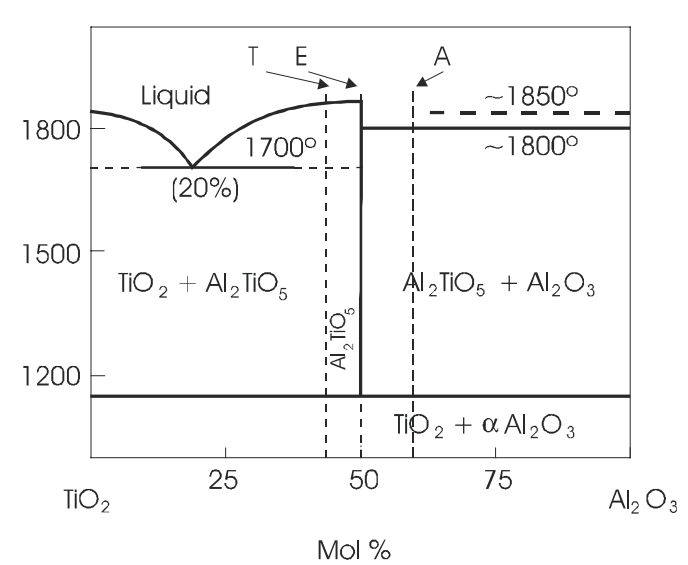

Figura 1: Diagrama de Equilibrio $\mathrm{Al}_{2} \mathrm{O}_{3}-\mathrm{TiO}_{2}$ [6].

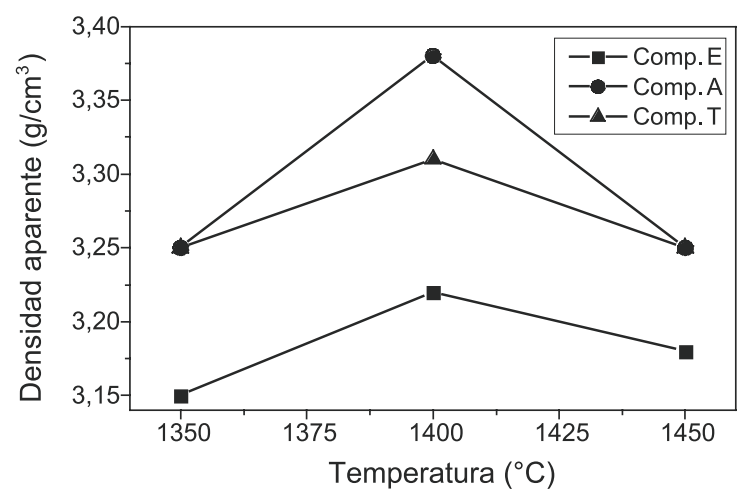

Figura 2: Densidad aparente para composiciones E, A y T.

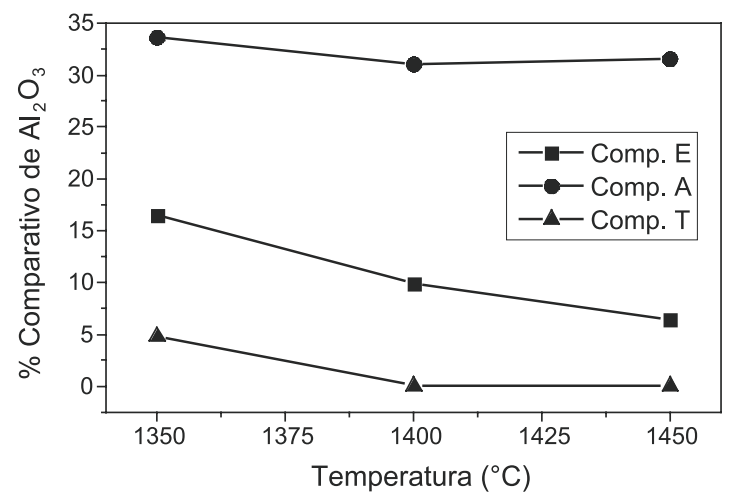

Figura 3: Porcentaje porcentual comparativa de la fase Alúmina sin reaccionar para composiciones $\mathrm{E}, \mathrm{A}$ y $\mathrm{T}$, sinterizadas reactivamente a 1350,1400 y $1450^{\circ} \mathrm{C}$

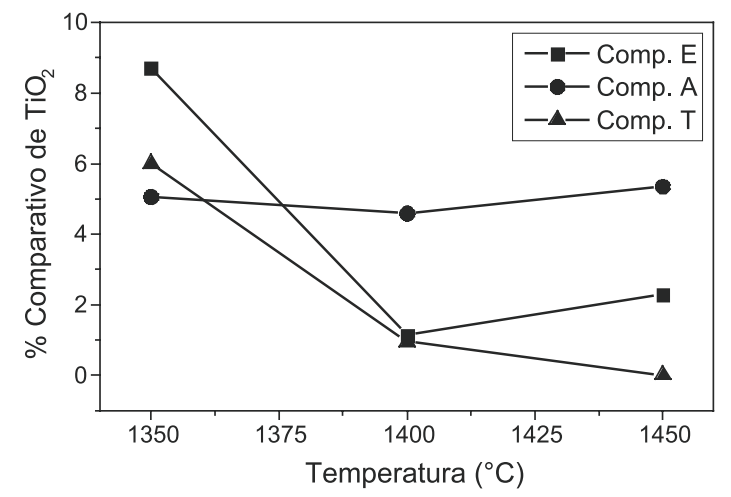

Figura 4: Análisis porcentual comparativo de la fase Óxido de Titanio sin reaccionar para las composiciones E, A y T, tratadas a 1350, $1400 \mathrm{y}$ $1450^{\circ} \mathrm{C}$. 


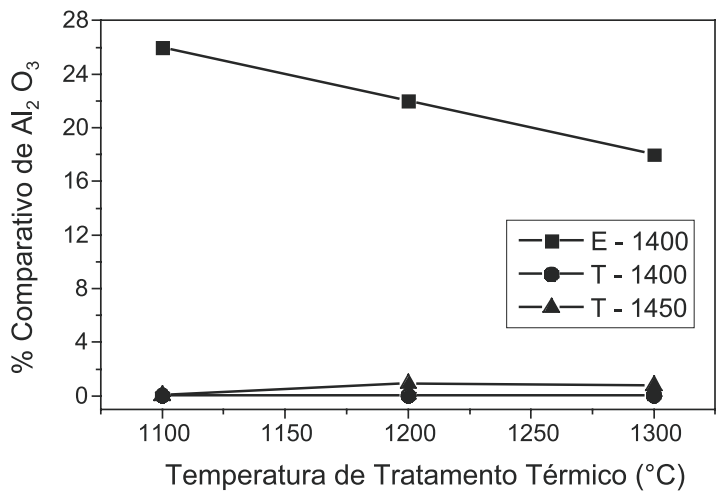

Figura 5: Análisis porcentual comparativo de la fase Alúmina encontrada después del tratamiento térmico de descomposición.

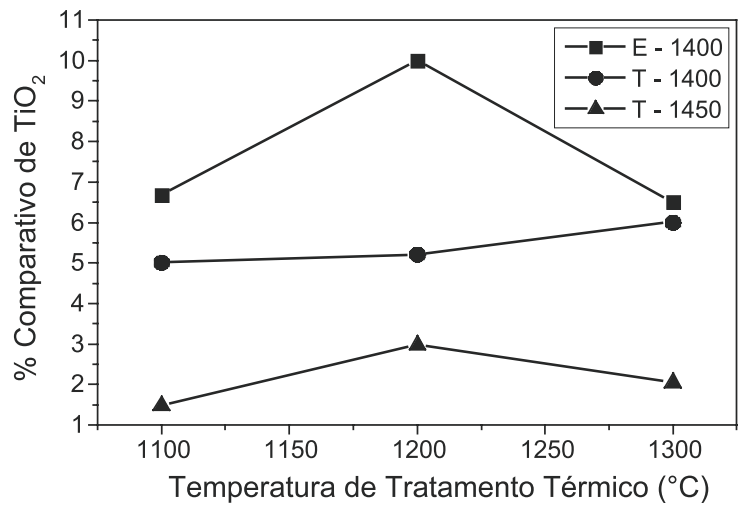

Figura 6: Análisis porcentual comparativo de la fase Óxido de Titanio encontrada después del tratamiento térmico de descomposición.

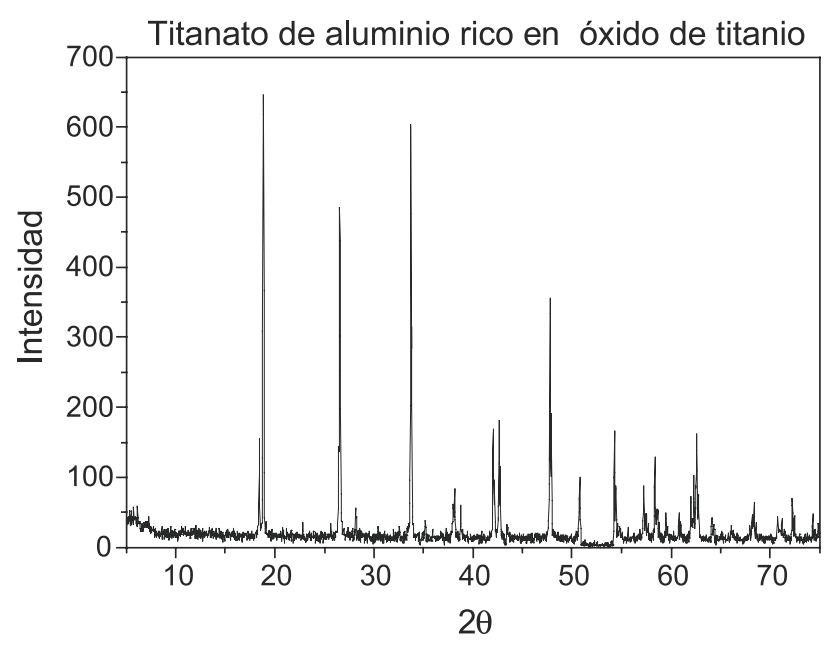

Figura 7: Difractograma de la composición $\mathrm{T}$ (excesso de Óxido de Titanio) cocido a $1400^{\circ} \mathrm{C}$. Todos los picos pertenecen a la fase $\mathrm{Al}_{2} \mathrm{TiO}_{5}$. que la formación de la fase $\mathrm{Al}_{2} \mathrm{TiO}_{5}$ sea completa (composición T). Una explicación probable para que la composición T se encuentre completamente reaccionada a $1450^{\circ} \mathrm{C}$ es que los iones titanio se encuentren en los interstícios cristalinos del titanato de aluminio formando una solución sólida.

El análisis comparativo porcentual de las fases encontradas después del tratamiento térmico de descomposición, usado para la investigación de la estabilidad del $\mathrm{Al}_{2} \mathrm{TiO}_{5}$, confirma los datos encontrados en la literatura [Ishitsuka et al., 1987], pués las partículas de Alúmina residual actúan como núcleos para la descomposición, como se observó en la composición estequiométrica, sinterizada a $1400^{\circ} \mathrm{C}$ (E-1400) después del tratamiento térmico. Existe un aumento del \% de Alúmina y Óxido de Titanio libres.

Se puede observar también que el Óxido de Titanio residual aumenta la estabilidad térmica del $\mathrm{Al}_{2} \mathrm{TiO}_{5}$. Esto ocurre probablemente porque el $\mathrm{TiO}_{2}$ se recombina con la $\mathrm{Al}_{2} \mathrm{O}_{3}$, ya que después del tratamiento térmico entre 1100 y $1300^{\circ} \mathrm{C}$ no se observó la presencia de $\mathrm{Al}_{2} \mathrm{O}_{3}$ en la composición T-1400. Sin embargo en la composición T-1450 (donde no se observó Óxido de Titanio residual) después del tratamiento térmico de descomposición, solamente ha encontrado un pequeño porcentaje de Alúmina $(<1 \%)$.

Con relación a la descomposición, de las Figuras 5 y 6 se puede concluir que la temperatura crítica de descomposición para las tres muestras tiene lugar en torno a $1100-1200^{\circ} \mathrm{C}$. A temperaturas de tratamiento térmico superiores, el Titanato de Aluminio se torna estable [7], mostrado por la disminución del porcentaje de los óxidos $\mathrm{Al}_{2} \mathrm{O}_{3}$ y $\mathrm{TiO}_{2}$.

Las muestras con exceso de Óxido de Titanio después del tratamiento térmico de descomposición presentaron niveles más elevados de Óxido de Titanio, probablemente porque el titanio que había entrado en el retículo cristalino del titanato de aluminio, formando un titanato rico en Óxido de Titanio, a temperaturas menores a la de sinterización, abandonó la red.

En la Figura 7 notamos que el exceso de Óxido de Titanio favorece la obtención de compactos de $\mathrm{Al}_{2} \mathrm{TiO}_{5}$ de elevada pureza, la aparente ausencia de $\mathrm{TiO}_{2}$ puede ser debido a los límites de resolución de la difracción de rayos-x o a la posible formación de una solución sólida de $\mathrm{TiO}_{2}$ en $\mathrm{Al}_{2} \mathrm{TiO}_{5}$.

\section{CONCLUSIONES}

- Un exceso de Óxido de Titanio $\left(\mathrm{TiO}_{2}\right)$ da lugar a materiales de titanato de aluminio, sin la presencia de otras fases después del processo de sinterización reactiva a $1450^{\circ} \mathrm{C}$.

- La composición estequiométrica genera materiales con Alúmina sin reaccionar a $1450^{\circ} \mathrm{C}$.

- Las composiciones con Óxido de Titanio sin reaccionar se mostraron más estables después de tratamientos térmicos en el ranjo de $800-1300^{\circ} \mathrm{C}$. Esto puede indicar que los núcleos de Alúmina son potencialmente más dañinos para la desestabilización del $\mathrm{Al}_{2} \mathrm{TiO}_{5}$ que los núcleos de $\mathrm{TiO}_{2}$

- La presencia de Alúmina residual origina la descomposición del titanato formado en Óxido de Titanio y Alúmina.

- Se há observado una mayor densificación en todas las composiciones sinterizadas a $1400^{\circ} \mathrm{C}$, probablemente porque por encima de esta temperatura hay un aumento del tamaño de grano de la fase Titanato de Aluminio, provocando un aumento de la densidad de microgrietas. 


\section{REFERENCIAS BIBLIOGRÁFICAS}

1. M. Ishitsuka, T. Sato, T. Endo, M. Shimada. Synthesis and Thermal Stability of Aluminum Titanate Solid Solutions. J. Am. Ceram. Soc., 70 [2], 69-71, (1987).

2. P. Jeschke, I. Elstner, D. Grimm, S. Pischek. Aluminium Titanate: a New Material for the Non-Ferrous Metal Industry. Unitecr'93 Congress, São Paulo, Brasil, 843-853,(1993).

3. D.S. Perera, R.L. Jeffries, D.C. Pender. Fabrication of Aluminium Titanate for Refractory Applications in Non-Ferrous Metals Industry. Unitecr'93 Congress, São Paulo, Brasil, 877-888, (1993).
4. D.S. Perera. Reaction-Sintered Aluminium Titanate. Journal of Material Science Letters, 8, 1057-1059, (1989).

5. H.A. Thomas, R. Stevens. Aluminium Titanate - A Literature Review, Part 1 Microcracking Phenomena. Br. Ceram. Trans. J., 88, 144-151, (1989).

6. E.M. Levin, H.F. McMurdie. Phase Diagrams for Ceramists, vol. III. Edited by The American Ceramic Society,INC. Fig.4376, 135, (1975).

7. E. Kato, K. Daimon, J. Takahashi. Decomposition temperature of $\beta-\mathrm{Al}_{2} \mathrm{TiO}_{5}$. J. Am. Ceram. Soc., 63 [5-6], 355-356, (1980).

Recibido: 18.02 .02

Aceptado: 26.11 .02

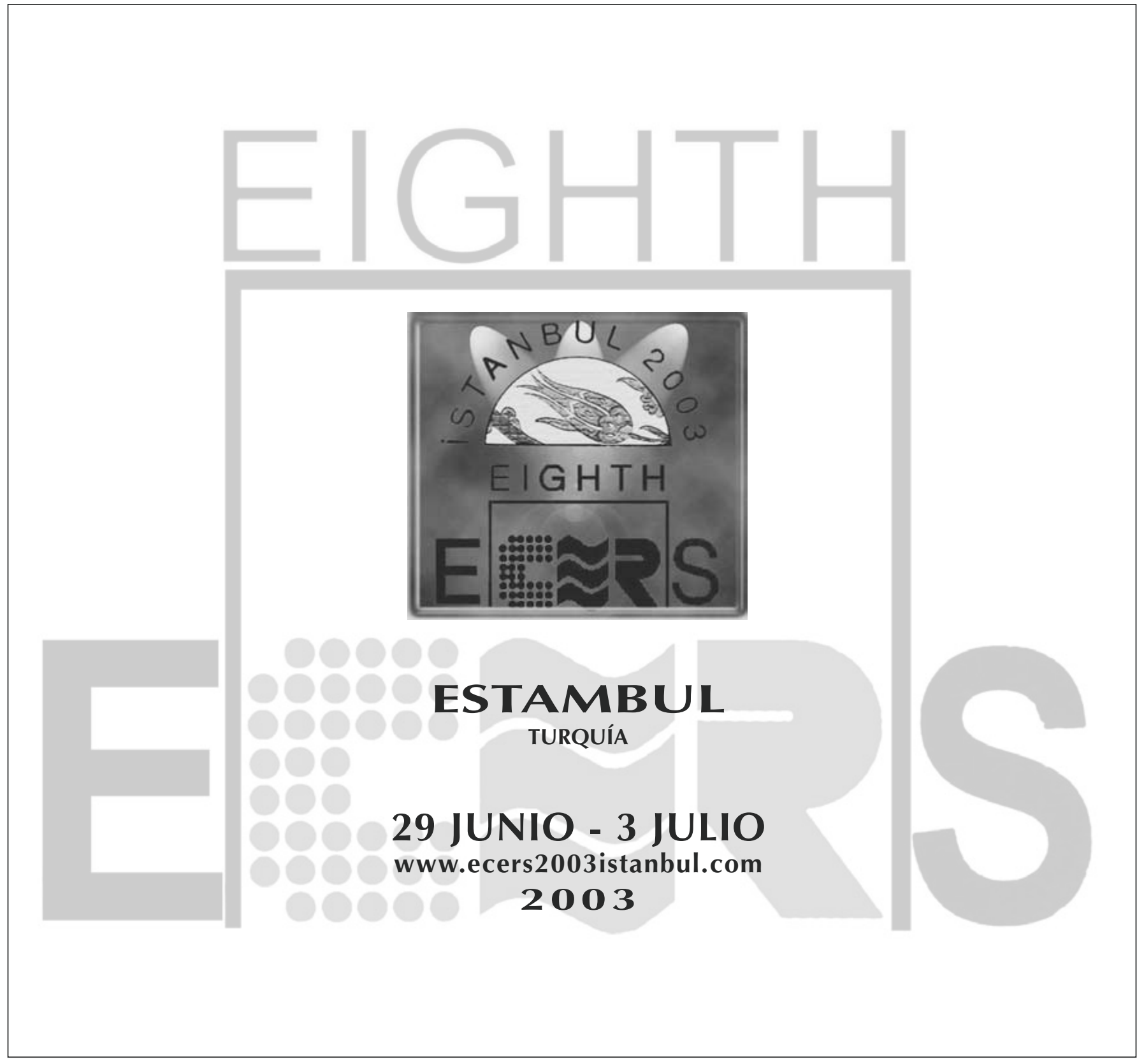

